\title{
CLINICO-EPIDEMIOLOGICAL PROFILE OF PATIENTS WITH PSORIASIS IN A TERTIARY CARE HOSPITAL OF WESTERN NEPAL
}

\author{
Aruna Sayami, ${ }^{1}$ Anita Shah, ${ }^{2}$ Yogesh Poudyal $^{3}$
}

\begin{abstract}
INTRODUCTION

Psoriasis is a common, chronic, inflammatory and proliferative condition of the skin which poses a significant health problem in general population. It is clinically characterized by erythematous plaques covered by silvery white scales, associated with systemic manifestations in many organ systems.
\end{abstract}

\section{MATERIAL AND METHODS}

This is a descriptive study conducted at the Department of Dermatology, Universal College of Medical Sciences, Bhairahawa, Nepal. All psoriasis patients who gave consent were clinically examined and proforma documentation including patient's clinical and demographic details was studied.

\section{RESULTS}

Fifty two psoriasis patients were studied. The median age was 40 years and male: female ratio was 1.7:1 with 33 (63.5\%) male and $19(36.5 \%)$ female psoriasis patients. The most common clinical type of psoriasis observed according to morphology was chronic plaque psoriasis $45(86.54 \%)$ and according to involvement of anatomical site was nail psoriasis $33(63.5 \%)$. This study showed that $30(57.7 \%)$ had no symptoms of pruritus and pain and $29(55.8 \%)$ of psoriatic cases had seasonal variation. The commonest treatment modality was a combination of oral and topical medication 29 (55.8\%).

\section{CONCLUSION}

Psoriasis is a common skin disorder associated with systemic manifestations seen by dermatologists. This study outlines the clinico-epidemiological profile of patients with psoriasis which can provide further guidance for taking appropriate measures in diagnosing, providing adequate treatment, and undertaking various preventive measures among psoriasis patients.

\section{KEYWORDS}

Clinical symptoms, Epidemiological profile, Psoriasis.

1 Department of Dermatology, Institute of Medicine, Tribhuwan University, Kathmandu

2 Department of Pathology, Universal College of Medical Sciences, Bhairahawa

3 Sarnath Skin Centre, Shantipath-8, Bhairahawa

DOI: https://doi.org/10.3126/jucms.v9i02.42003

For Correspondence

Dr. Aruna Sayami

Department of Dermatology

Institute of Medicine (IOM)

Tribhuwan University.

E-mail: sayamiaruna@gmail.com 


\section{INTRODUCTION}

Psoriasis is characterized by red, scaly, sharply demarcated, indurated plaques present particularly over the extensor surfaces and scalp, affecting 2 to $4 \%$ of the population. ${ }^{1,2}$ The clinical classification of psoriasis according to age of onset are Type I psoriasis with age of onset before 40 years, is often associated with positive family history, and Type II psoriasis with age of onset after 40 years. ${ }^{3,4}$ The classification according to morphology are chronic plaque psoriasis, guttate psoriasis, erythrodermic psoriasis, pustular psoriasis (generalized/ localized), rupioid psoriasis, elephantine psoriasis; according to psoriasis affecting specific sites are scalp, palmoplantar, genital, nail, inverse/flexor and seborrheic psoriasis. ${ }^{5}$

There are various risk factors associated with disease causation and exacerbation including alcohol, smoking, psychogenic factors (stress), sunlight, obesity, low physical activity and metabolic syndrome including diabetes mellitus type 2, hypertension. ${ }^{1,6}$ For less severe disease topical treatment, phototherapy is a common treatment modality. For severe disease oral medication including methotrexate, cyclosporine, apremilast are the first line of management. ${ }^{1}$

Psoriasis is one of the common, chronic skin diseases seen by dermatologists associated with systemic manifestations. Patients seek consultation from multitude of specialists, resulting in frustration in the patient and the treating specialist. This study enables us to determine the demographic profile, clinical characteristics, symptoms, exacerbating factors and common treatment modalities among patients with psoriasis in context to the Western Terai population. Therefore, this study aimed to understand the current scenario about this disease which may further aid in early diagnosis and proper management of the disease.

\section{MATERIALAND METHODS}

This descriptive study was conducted in Department of Dermatology, Universal College of Medical Sciences (UCMS), Bhairahawa, Nepal from January to December 2017. All patients with psoriasis attending the Dermatology outpatient department (OPD) of UCMS, Bhairahawa, who gave consent for clinical examination was included in the study. Patients/guardians who did not give consent because of any reason were excluded.

Before conducting the study, the proposal was submitted to the Institutional Review Board (IRB) of UCMS and the ethical clearance was taken. The will of the participants were fully respected and a written consent was taken after fully explaining all the relevant details, its importance and implications. Confidentiality of data was maintained. We enrolled fifty two psoriasis patients meeting the inclusion criteria. Consecutive sampling technique was used. After proper counselling, clinical examination with proforma documentation including patient details, examination findings were performed.
The data were entered in Microsoft Excel and transferred to IBM - Statistical Package for Social Sciences (SPSS) version 20. We calculated frequencies and percentage of the cases.

\section{RESULTS}

The age range of psoriasis patients in the study spanned from 11 to 80 years. The maximum number of patients belonged to age group of 31-40 years with $15(28.8 \%)$ followed by age group of 51-60 years with $11(21.2 \%)$ psoriasis patients with the median age of 40 years. The distribution according to gender showed $33(63.5 \%)$ male and 19 (36.5\%) female psoriasis patients. The male:female ratio was approximately 1.7:1. The distribution of patients according to religion showed $50(96.2 \%)$ patients belonging to Hindu religion and 2 (3.8\%) patients belonging to Muslim religion.

Out of 52 patients, there were 21 (40.4\%) smokers, 22 (42.3\%) consumed alcohol, $49(94.2 \%)$ were non-vegetarian and only $2(3.8 \%)$ patients gave history of diabetes mellitus and hypertension respectively. $29(55.8 \%)$ were taking oral and topical medication and $11(21.2 \%)$ were under only topical medication. $48(92.3 \%)$ had no any family history of the same disease whereas remaining $4(7.7 \%)$ gave significant positive history of psoriasis in family (Table 1).

Table 1. Distribution of psoriasis patients according to the demographic details and history

\begin{tabular}{lc}
\hline Age groups (years) & Psoriasis n (\%) \\
\hline $11-20$ & $6(11.5 \%)$ \\
$21-30$ & $6(11.5 \%)$ \\
$31-40$ & $15(28.8 \%)$ \\
$41-50$ & $7(13.5 \%)$ \\
$51-60$ & $11(21.2 \%)$ \\
$61-70$ & $4(7.7 \%)$ \\
$71-80$ & $3(5.8 \%)$ \\
Gender & \\
Male & $33(63.5 \%)$ \\
Female & $19(36.5 \%)$ \\
Religion & \\
Hindu & $50(96.2 \%)$ \\
Muslims & $2(3.8 \%)$ \\
Smoking & \\
Present & $21(40.4 \%)$ \\
Absent & $31(59.6 \%)$ \\
Alcohol drinking & \\
Present & $22(42.3 \%)$ \\
Absent & $30(57.7 \%)$ \\
Dietary habit & \\
Vegetarian & $3(5.8 \%)$ \\
Non-vegetarian & $49(94.2 \%)$ \\
Diabetes mellitus & \\
Present & $2(3.8 \%)$ \\
Absent & $50(96.2 \%)$ \\
Hypertension & $2(3.8 \%)$ \\
Present & $50(96.2 \%)$ \\
Absent &
\end{tabular}


The most common clinical type of psoriasis observed was chronic plaque psoriasis $45(86.54 \%)$, followed by palmoplantar psoriasis 4 (7.69\%), guttate psoriasis, pustular psoriasis and scalp psoriasis comprising of $1(1.92 \%)$ of total psoriasis patients respectively. (Figure 1).

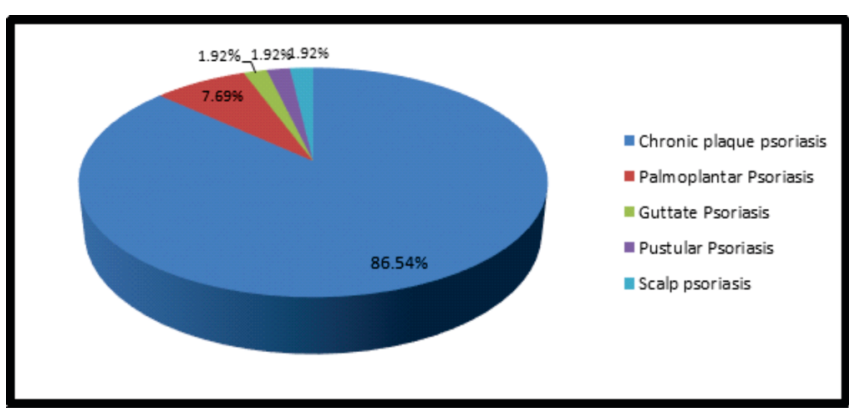

Figure 1. Distribution of psoriasis patients according to its different morphological forms

Out of the 52 patients, $30(57.7 \%)$ had no symptoms of pruritus and pain, $16(30.7 \%)$ complained of pruritus and 6 (11.5\%) complained of pruritus and pain (Figure 2).

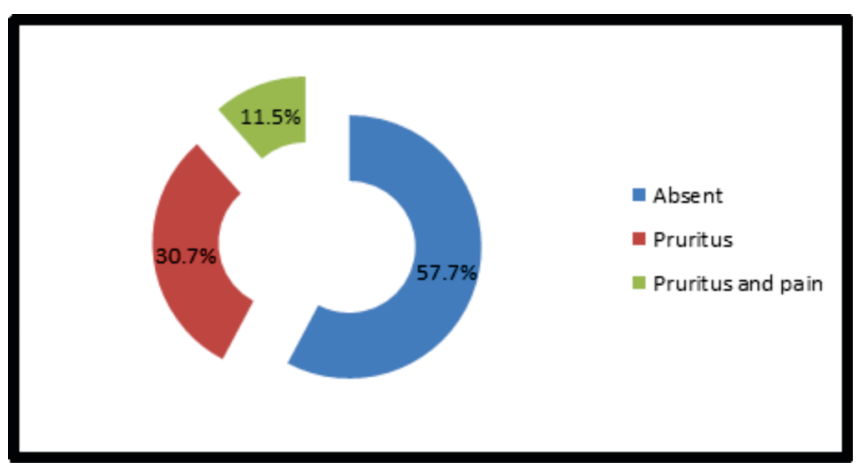

Figure 2. Distribution of psoriasis patients according to symptoms

According to involvement of anatomical site, it was observed that nail psoriasis was commonest 33 (63.5\%), followed by involvement of flexural/inverse psoriasis in $30(57.7 \%)$ patients (Figure 3).

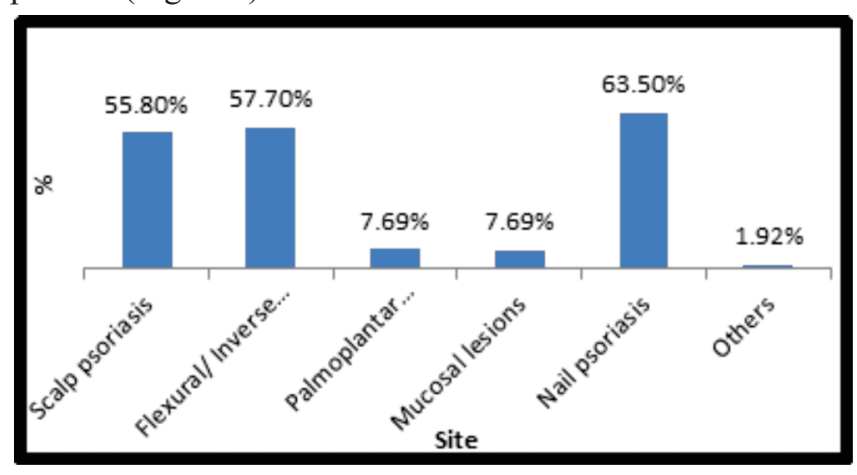

Figure 3. Distribution of psoriasis according to site of lesion
The distribution of psoriasis according to the duration of lesions showed $20(38.6 \%)$ patients had disease duration of less than six months and $17(32.69 \%)$ showed duration of more than two years. Among 52 psoriasis patients, 29 (55.8\%) patients showed seasonal variation in the form of exacerbation of the lesions in winter whereas $23(44.2 \%)$ patients had no seasonal variation of disease.

\section{DISCUSSION}

Psoriasis is most likely to appear between the ages of 15 and 30 years but ranges from birth to the eighth or ninth decade with bimodal distribution. ${ }^{1}$ In our study the maximum number of patients belonged to age group of 31-40 years followed by 51-60 years in psoriasis patients. One study in United Kingdom showed bimodal distribution of disease, the peak being 16-22 years and 57-60 years. Our study showed that the median age of 40 years. Likewise, in a study done by Solak et $\mathrm{al}^{8}$ the median age was 40 years. Another study by Das et al showed mean age of $39.7 \pm 7.3$ years among psoriasis group. ${ }^{9}$

In this study, the male patients were predominant comprising of $63.5 \%$ of study patients $(n=33)$ and female patients were $36.5 \%(\mathrm{n}=19)$. The male predominance was also noted in a study done by Sampogna et al. ${ }^{10}$ The male to female ratio was approximately 1.7:1 in our study which was in accordance to a study done in Japan where the ratio was 1.98:1 done by Takahashi et al. ${ }^{11}$

In our study, we found that $40.4 \%$ of psoriasis patients were smokers. The research of Heron et $\mathrm{al}^{12}$ showed that smoking is associated with the onset of psoriasis. Our study showed that $42.3 \%$ of psoriatic patients consumed alcohol. Likewise, Qureshi et $\mathrm{al}^{13}$ had mentioned that alcohol intake was associated with relative risk of 1.72 for acquiring psoriasis. The study also showed alcohol was not only associated with the disease after the onset of disease, but alcohol intake itself leads to exacerbation of the disease. Our study showed that diabetes and hypertension was present only among two $(3.8 \%)$ psoriatic patients respectively. However, Langan et $\mathrm{al}^{14}$ showed associations of metabolic diseases including diabetes with psoriasis. The study of Neimann et $\mathrm{al}^{15}$ has shown that hypertension is 1.16 times more prevalent in psoriasis in comparison to general population. These findings suggest that screening for metabolic disease should be considered in psoriasis patients.

In our study, $29(55.8 \%)$ were taking oral and topical medication and $11(21.2 \%)$ were under only topical medication. Similarly, Takahashi et $\mathrm{al}^{11}$ showed topical corticosteroids $(78.1 \%)$ and vitamin D3 (18.1\%) products were the main previous topical agents. Our study showed only $7.7 \%$ of patients had significant family history. However, a study done by Solak et $\mathrm{al}^{8}$ showed family history as a risk factor for psoriasis.

The most common clinical type of psoriasis observed was chronic plaque psoriasis $45(86.54 \%)$, followed by palmo- 
plantar psoriasis 4 (7.69\%). This was similar with study done in Japan by Takahasi et $\mathrm{al}^{11}$ which showed the commonest clinical type of psoriasis to be psoriasis vulgaris (91.5\%). Chronic plaque psoriasis was also the commonest diagnosis in a study done by Sampogna et al, ${ }^{10}$ and Kawada et al. ${ }^{16}$

In our study $30(57.7 \%)$ had no symptoms of pruritus and pain, $16(30.7 \%)$ complained of pruritus and $6(11.5 \%)$ complained of pruritus and pain. Likewise, Kwon et $\mathrm{al}^{17}$ showed in elderlyonset patients, $32(25.4 \%)$ experienced no symptom of pruritus around psoriatic lesions, while $50(39.7 \%)$ patients complained about mild symptoms.

According to involvement of anatomical site, our study showed nail psoriasis was present in 33 (63.5\%), followed by involvement of flexural/inverse psoriasis in $30(57.7 \%)$ patients, scalp in $29(55.8 \%)$. In contrast, Kwon et al ${ }^{17}$ showed in elderly-onset patients, scalp $(36.8 \%)$ was the most frequent site, followed by nail $(27.9 \%)$. Solak et $\mathrm{al}^{8}$ showed involvement of nails in $60(30.3 \%)$ of psoriatic patients. This study showed $29(55.8 \%)$ of psoriatic cases had seasonal variation. All these cases showed the exacerbation of disease occurring in winter. This resembled to the study done by Takahashi et al, ${ }^{11}$ Kawada et al, ${ }^{16}$ which supports the fact that psoriasis is exacerbated in winter showing seasonal variation.

\section{CONCLUSION}

Psoriasis was more common in male population. The commonest clinical type was chronic plaque psoriasis and the commonest treatment modality was a combination of oral and topical medication among patient visiting the tertiary center. Our study can provide further guidance for taking appropriate measures in diagnosing, providing adequate treatment, and undertaking various preventive measures among psoriasis patients.

\section{ACKNOWLEDGEMENTS}

My sincere thanks to Mrs. Jamuna Tamrakar Sayami, Mr. Narayan Gautam and Dr. Megha Raj Banjara for reviewing this manuscript.

\section{CONFLICT OF INTEREST}

None

\section{REFERENCES}

1. Griffiths EMC, Barker J, Bleiker T, Chalmers R CD. Rook's Textbook of Dermatology. 9th ed. Vol. 2, Psoriasis and Related Disorders. Wiley Blackwell; 2016;35.1-35.48.

2. Griffiths CE, Barker JN. Pathogenesis and clinical features of psoriasis. Lancet. 2007;370(9583):263-71.

3. Agwu B, Agwu FMCP B, Perpetua Uchechi I. Hyperuricemia in Nigerian Psoriatic Patients. Quest Journals J Med Dent Sci Res. 2016;3(4):12-6.
4. Gudjonsson JE, Elder JT. Psoriasis: epidemiology. Clin Dermatol. 2007;25(6):535-46.

5. Raychaudhuri SK, Maverakis E, Raychaudhuri SP. Diagnosis and classification of psoriasis. Autoimmun Rev. 2014;13 (4-5):490-5.

6. Herron MD, Hinckley M, Hoffman MS, Papenfuss J, Hansen $\mathrm{CB}$, Callis KP, et al. Impact of obesity and smoking on psoriasis presentation and management. Arch Dermatol. 2005;141 (12):1527-34

7. Smith AE, Kassab JY, Rowland Payne CM, Beer WE. Bimodality in age of onset of psoriasis, in both patients and their relatives. Dermatology. 1993;186(3):181-6.

8. Solak B, Dikicier BS, Erdem T. Impact of elevated serum uric acid levels on systemic inflammation in patients with psoriasis 2017;68(3):266-70.

9. Das S, Biswas UK, Kumar A, Roy A study of serum carbonic anhydrase activity, uric acid, C-reactive protein levels and lipid parameters in patients with Psoriasis. 2013;11(1).

10. Sampogna F, Gisondi P, Melchi CF, Amerio P, Girolomoni G, Abeni D. Prevalence of symptoms experienced by patients with different clinical types of psoriasis. Br J Dermatol. 2004;151(3):594-9.

11. Takahashi H, Takahashi I, Tsuji H, Ibe M, Kinouchi M, Hashimoto Y, et al. Analysis of psoriatic patients registered in Asahikawa Medical College Hospital from 1983 to 2007. J Dermatol. 2009;36(12):632-7.

12. Herron MD, Hinckley M, Hoffman MS, Papenfuss J, Hansen $\mathrm{CB}$, Callis KP, et al. Impact of obesity and smoking on psoriasis presentation and management. Arch Dermatol. 2005;141(12) $: 1527-34$

13. Qureshi AA, Dominguez PL, Choi HK, Han J, Curhan G. Alcohol intake and risk of incident psoriasis in US women: A prospective study. Arch Dermatol. 2010;146(12):1364-9.

14. Langan SM, Seminara NM, Shin DB, Troxel AB, Kimmel SE, Mehta NN, et al. Prevalence of metabolic syndrome in patients with psoriasis: A population-based study in the United Kingdom. Vol. 132, Journal of Investigative Dermatology. 2012. Mar;132(3 Pt 1):556-62.

15. Neimann AL, Shin DB, Wang X, Margolis DJ, Troxel AB GJ. Prevalence of cardiovascular risk factors in patients with psoriasis. JAm Acad Dermatol. 2006;55:829-35.

16. Kawada A, Tezuka T, Nakamizo Y, Kimura H, Nakagawa $H$ Ohkido M, et al. A survey of psoriasis patients in Japan from 1982 to 2001. J Dermatol Sci. 2003;31(1):59-64.

17. Kwon HH, Kwon IH, Youn J Il. Clinical study of psoriasis occurring over the age of 60 years: Is elderly-onset psoriasis a distinct subtype? Int J Dermatol. 2012;51(1):53-8. 